\title{
Analytical solution of a two-dimensional elastostatic problem of functionally graded materials via the Airy stress function
}

\author{
H. Sakurai \\ Co-operative Education Centre, \\ Sendai National College of Technology, Japan
}

\begin{abstract}
Functionally Graded Materials (FGMs) possess properties that vary gradually as a function of spatial coordinates. They are different from conventional composite materials in that they have no distinct interfaces at which their material properties change abruptly. These FGMs are suitable for various applications, such as aerospace, nuclear fusion, biomaterial electronics, etc. In practice, applications of analytical solutions are limited. However, the analytical solutions are very important as standards for evaluating numerical simulation results and they are also important to mathematical understanding. Little research on the analytical solutions of two-dimensional elastostatic problems has been reported. Furthermore, few analytical solutions using Airy stress functions have been published. The purpose of this paper is to propose an analytical method for the two-dimensional elastostatic problems of FGMs using the Airy stress function. In the present investigation, FGMs in which the properties of the materials vary exponentially in one direction are examined. A few numerical examples are presented and the validity of the method is shown by comparisons with the results of past studies.

Keywords: analytical solution, functionally graded material, two-dimensional problem, Airy stress function.
\end{abstract}

\section{Introduction}

The Functionally Graded Materials (FGMs) possess properties that vary gradually as a function of spatial coordinates. They are different from conventional composite materials in that they have no distinct interfaces at which 
their material properties change abruptly [1]. These FGMs are suitable for various applications, such as aerospace, nuclear fusion, biomaterial electronics, etc. Chakraborty et al. developed a new beam element solving FGM beam structures based on the first order shear deformation theory [2]. Nguyen et al. proposed a first-order shear deformation plates models for modeling structures made of FGMs [3]. Xinag and Yang studied free and forced vibrations of a laminated FGM Timoshenko beam with variable thickness under heat conduction [4]. Sankar and his co-workers have been reporting analytical methods for the thermomechanical analysis of FGM beams [5-7].

Zhu and Sankar presented an elasticity solution of a simply supported FGM beam having variation of Young's modulus distributed by a polynomial in the thickness direction [6]. In Ref. [6], the Fourier series method is used to reduce the governing partial differential equations to the ordinary equations that are then solved by the Galerkin method. Miers and Telles proposed the Boundary Element-Free Method belonging to a meshless technique, for two-dimensional elastostatic analysis of FGMs [8]. Zhong and Yu presented explicit solutions of a cantilever FGM beam having arbitrary graded variations of material properties distributed in the thickness direction based on two-dimensional theory of elasticity [9].

Applications of analytical solutions are limited to practical shapes of analysis regions and boundary conditions. However, the analytical solutions are very important as standards for evaluating numerical simulation results, such as finite element method etc., and they are also important to mathematical understanding. Little research on the analytical solutions of two-dimensional elastostatic problems has been reported. Furthermore, to the best of the author's knowledge, few analytical solutions using Airy stress functions have been published.

The objective of the present paper is to describe the analysis of the twodimensional elastostatic problems of FGMs using the Airy stress function. In this study, FGMs in which the properties of the materials change exponentially in one direction are treated. A few numerical examples are presented and the validity of the method is shown by the comparisons with the results of the present method and results of past studies.

\section{Two-dimensional problem of FGMs and basic equations}

In the Cartesian coordinate system $O-x z$, we consider a simply supported FGM beam subjected to a transverse load as shown in Fig. 1. The length in the $x$ direction is $l$ and the length in the $z$ direction is $h$. In Fig. 1, the uniform transverse load and the simply supported boundary condition are one of the examples.

In the absence of body forces the equilibrium equations are given as

$$
\frac{\partial \sigma_{x x}}{\partial x}+\frac{\partial \sigma_{z x}}{\partial z}=0 \quad \frac{\partial \sigma_{x z}}{\partial x}+\frac{\partial \sigma_{z z}}{\partial z}=0
$$

where $\sigma_{x x}, \sigma_{z z}, \sigma_{z x}$ are stress components and $\sigma_{z x}=\sigma_{x z}$. 


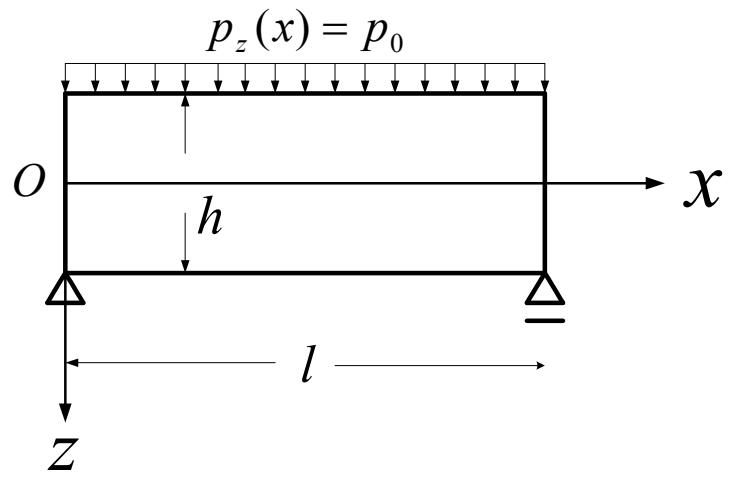

Figure 1: An example of a simply supported FGM beam.

The relationships between strains and displacements are

$$
\varepsilon_{x x}=\frac{\partial u}{\partial x} \quad \varepsilon_{z z}=\frac{\partial w}{\partial z} \quad \varepsilon_{z x}=\frac{\partial u}{\partial z}+\frac{\partial w}{\partial x}
$$

where $\varepsilon_{x x}, \varepsilon_{z z}, \varepsilon_{z x}$ are strain components and $u, w$ are the displacement components in the $x$ and the $z$ direction respectively. The strain components should also satisfy the following compatibility condition.

$$
\frac{\partial^{2} \varepsilon_{x x}}{\partial z^{2}}+\frac{\partial^{2} \varepsilon_{z z}}{\partial x^{2}}-\frac{\partial^{2} \varepsilon_{z x}}{\partial z \partial x}=0
$$

The constitutive equations are given as

$$
\varepsilon_{x x}=s_{11} \sigma_{x x}+s_{13} \sigma_{z z} \quad \varepsilon_{z z}=s_{13} \sigma_{x x}+s_{33} \sigma_{z z} \quad \varepsilon_{z x}=s_{44} \sigma_{z x}
$$

where $s_{11}, s_{13}, s_{33}, s_{44}$ are elastic moduli. The material properties of FGMs change gradually as a function of spatial coordinates. We assume material properties varying exponentially in the $z$ direction, i.e.

$$
s_{i j}=s_{i j}^{0} \cdot F(z)=s_{i j}^{0} \cdot e^{\frac{\alpha z}{h}}
$$

where $S_{i j}^{0}$ are their corresponding values in the plane $z=z_{0}$ with $F\left(z_{0}\right)=1$ and $F(z)$, is called graded function, which expresses the distribution of material properties, and the parameter $\alpha$ is called the graded index [9]. Now, we introduce Airy stress function $\Phi(x, z)$ expressed by the following equations.

$$
\sigma_{x x}=\frac{\partial^{2} \Phi}{\partial z^{2}} \quad \sigma_{z z}=\frac{\partial^{2} \Phi}{\partial x^{2}} \quad \sigma_{z x}=-\frac{\partial^{2} \Phi}{\partial z \partial x}
$$


Substituting Eqns. (4)-(6) into Eqn. (3), the next governing equation with respect to Airy stress function $\Phi(x, z)$ is obtained.

$$
\begin{aligned}
& s_{11}^{0} \frac{\partial^{4} \Phi}{\partial z^{4}}+\left(2 s_{13}^{0}+s_{44}^{0}\right) \frac{\partial^{4} \Phi}{\partial x^{2} \partial z^{2}}+s_{33}^{0} \frac{\partial^{4} \Phi}{\partial x^{4}}+2 s_{11}^{0} \frac{\alpha}{h} \frac{\partial^{3} \Phi}{\partial z^{3}} \\
& +\left(2 s_{13}^{0}+s_{44}^{0}\right) \frac{\alpha}{h} \frac{\partial^{3} \Phi}{\partial x^{2} \partial z}+s_{11}^{0} \frac{\alpha^{2}}{h^{2}} \frac{\partial^{2} \Phi}{\partial z^{2}}+s_{13}^{0} \frac{\alpha^{2}}{h^{2}} \frac{\partial^{2} \Phi}{\partial x^{2}}=0
\end{aligned}
$$

The above equation is the same form as the governing equation of the plates bending with variable rigidity, and it is possible to adopt the same method for the solution [10].

\section{Method of solution}

In Eqn. (7), we introduce the following Airy stress function $\Phi(x, z)$.

$$
\Phi(x, z)=\sum_{i=1}^{\infty} A_{i}(z) \cdot f_{i}(x)
$$

The function $f_{i}(x)$ should be assumed to satisfy the mechanical boundary conditions at the $x=0$ and $x=l$. For instance, under the simply supported condition and the cantilever condition, it should be chosen to satisfy $\sigma_{x x}=0, \sigma_{z z}=0$ at the both ends and $\sigma_{x x}=0$ and $\sigma_{x z}=0$ at the free end respectively.

\subsection{Formulation for simply supported FGM beam subjected to uniform pressure}

In this problem, we assume the following function

$$
\Phi=\sum_{i=1}^{\infty} A_{i}(z) \cdot f_{s i}(x)=\sum_{i=1,3,5 \ldots}^{\infty} A_{i}(z) \cdot \sin \xi_{i} x
$$

where $\xi_{i}=\frac{i \pi}{l}, f_{s i}(x)=\sin \xi_{i} x$. And the stress components are as follows

$$
\begin{aligned}
\sigma_{x x} & =\sum_{i=1,3,5 \ldots}^{\infty} \frac{d^{2} A_{i}(z)}{d z^{2}} \cdot f_{s i}(x) \\
\sigma_{z z} & =\sum_{i=1,3,5 \cdots}^{\infty}-\xi_{i}^{2} A_{i}(z) \cdot f_{s i}(x) \\
\sigma_{z x} & =\sum_{i=1,3,5 \cdots}^{\infty}-\xi_{i} \frac{d A_{i}}{d z} f_{c i}(x)
\end{aligned}
$$


where $f_{c i}(x)=\cos \xi_{i} x$. Substituting Eqn. (9) into Eqn. (7), the following ordinary differential equation with respect to the unknown function $A_{i}(z)$ is obtained easily.

$$
\begin{aligned}
& s_{11}^{0} \frac{d^{4} A_{i}}{d z^{4}}+2 s_{11}^{0} \frac{\alpha}{h} \frac{d^{3} A_{i}}{d z^{3}}+\left\{s_{11}^{0} \frac{\alpha^{2}}{h^{2}}-\left(2 s_{13}^{0}+s_{44}^{0}\right) \xi_{i}^{2}\right\} \frac{d^{2} A_{i}}{d z^{2}} \\
& -\left(2 s_{13}^{0}+s_{44}^{0}\right) \xi_{i}^{2} \frac{\alpha}{h} \frac{d A_{i}}{d z}+\left(s_{33}^{0} \xi_{i}^{2}-s_{13}^{0} \frac{\alpha^{2}}{h^{2}}\right) \xi_{i}^{2} A_{i}=0
\end{aligned}
$$

The solution of the above equation is given as

$$
A_{i}(z)=C_{i, 1} e^{r_{i, 1} z}+C_{i, 2} e^{r_{i, 2} z}+C_{i, 3} e^{r_{i, 3} z}+C_{i, 4} e^{r_{i, 4} z}
$$

where the $r_{i, j}(j=1,2,3,4)$ are the roots of the following the 4-th order equation and the $C_{i, j}(j=1,2,3,4)$ are arbitrary constants to be determined by the boundary conditions at $z=-h / 2$ and $z=h / 2$.

$$
\begin{aligned}
& r_{i, j}{ }^{4}+2 \frac{\alpha}{h} r_{i, j}{ }^{3}+\left\{\frac{\alpha^{2}}{h^{2}}-\frac{1}{s_{11}^{0}}\left(2 s_{13}^{0}+s_{44}^{0}\right) \xi_{i}^{2}\right\} r_{i, j}{ }^{2} \\
& -\frac{1}{s_{11}^{0}}\left(2 s_{13}^{0}+s_{44}^{0}\right) \xi_{i}^{2} \frac{\alpha}{h} r_{i, j}+\frac{1}{s_{11}^{0}}\left(s_{33}^{0} \xi_{i}^{2}-s_{13}^{0} \frac{\alpha^{2}}{h^{2}}\right) \xi_{i}^{2}=0
\end{aligned}
$$

Considering Fourier expansion of the transverse pressure $p_{z}(x)=p_{0}$, the following equation is obtained.

$$
p_{z}=\frac{4 p_{0}}{\pi} \sum_{i=1,3,5 \ldots}^{\infty} \frac{1}{i} f_{s i}(x)=\frac{4 p_{0}}{\pi} \sum_{i=1,3,5 \ldots}^{\infty} \frac{1}{i} \sin \xi_{i} x
$$

The boundary conditions at the both surfaces are as follows.

$$
\begin{array}{ccc}
\sigma_{z z}=-p_{0} & , \sigma_{z x}=0 & \text { at } z=-h / 2 \\
\sigma_{z z}=0 & , \sigma_{z x}=0 & \text { at } z=h / 2
\end{array}
$$

Substituting Eqn. (10) and Eqn. (14) into Eqn. (15), the following four equations for determining the arbitrary constants $C_{i, j}$ are derived.

$$
\begin{array}{cc}
A_{i}\left(-\frac{h}{2}\right)=\frac{4 p_{0}}{\pi \xi_{i}^{2} i} & \frac{d A_{i}}{d z}\left(-\frac{h}{2}\right)=0 \\
A_{i}\left(\frac{h}{2}\right)=0 & \frac{d A_{i}}{d z}\left(\frac{h}{2}\right)=0
\end{array}
$$




\subsection{Formulation for cantilever FGM beam subjected to uniform pressure}

We deal with the formulation of a cantilever FGM beam such as shown in Fig. 2.

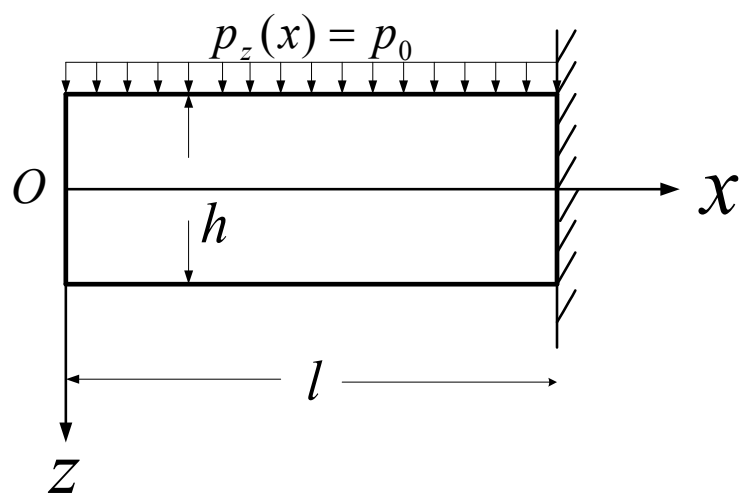

Figure 2: An example of a cantilever FGM beam.

It is necessary to assume the function $f_{i}(x)$ satisfying the mechanical boundary conditions at the free end. One of the simplest functions satisfying the above conditions is

$$
\Phi=A_{2}(z) \cdot f_{2}(x)=A_{2}(z) \cdot x^{2}
$$

where $f_{2}(x)=x^{2}$. The stress components are as follows.

$$
\begin{gathered}
\sigma_{x x}=\frac{\partial^{2} \Phi}{\partial z^{2}}=\frac{d^{2} A_{2}}{d z^{2}} \cdot f_{2}(x)=\frac{d^{2} A_{2}}{d z^{2}} \cdot x^{2} \\
\sigma_{z z}=\frac{\partial^{2} \Phi}{\partial x^{2}}=A_{2}(z) \cdot \frac{d^{2} f_{2}(x)}{d x^{2}}=A_{2}(z) \cdot 2 \\
\sigma_{z x}=-\frac{\partial^{2} \Phi}{\partial z \partial x}=-\frac{d A_{2}(z)}{d z} \cdot \frac{d f_{2}(x)}{d x}=-\frac{d A_{2}(z)}{d z} \cdot 2 x
\end{gathered}
$$

Substituting Eqn.(17) into Eqn.(7), the following ordinary differential equation is derived again.

$$
\begin{aligned}
& s_{11}^{0} x^{2} \frac{d^{4} A_{2}}{d z^{4}}+2 s_{11}^{0} \frac{\alpha}{h} x^{2} \frac{d^{3} A_{2}}{d z^{3}}+\left\{2\left(2 s_{13}^{0}+s_{44}^{0}\right)+s_{11}^{0} \frac{\alpha^{2}}{h^{2}} x^{2}\right\} \frac{d^{2} A_{2}}{d z^{2}} \\
& +2\left(2 s_{13}^{0}+s_{44}^{0}\right) \frac{\alpha}{h} \frac{d A_{2}}{d z}+2 s_{13}^{0} \frac{\alpha^{2}}{h^{2}} A_{2}=0
\end{aligned}
$$


We consider the above equation as the following ordinary differential equation with respect to $z$ at the given section $x=x_{0}$ (constant).

$$
\begin{aligned}
& s_{11}^{0} x_{0}{ }^{2} \frac{d^{4} A_{2}}{d z^{4}}+2 s_{11}^{0} \frac{\alpha}{h} x_{0}{ }^{2} \frac{d^{3} A_{2}}{d z^{3}}+\left\{2\left(2 s_{13}^{0}+s_{44}^{0}\right)+s_{11}^{0} \frac{\alpha^{2}}{h^{2}} x_{0}{ }^{2}\right\} \frac{d^{2} A_{2}}{d z^{2}} \\
& +2\left(2 s_{13}^{0}+s_{44}^{0}\right) \frac{\alpha}{h} \frac{d A_{2}}{d z}+2 s_{13}^{0} \frac{\alpha^{2}}{h^{2}} A_{2}=0
\end{aligned}
$$

The solution of the ordinary differential equation is given in the same form as Eqn. (12). The boundary conditions on the top $z=-h / 2$ and on the bottom $z=h / 2$ at the $x=x_{0}$ (constant) are expressed as follows.

$$
\begin{array}{ccc}
A_{2}\left(-\frac{h}{2}\right) \cdot 2=-p_{0} & \frac{d A_{2}}{d z}\left(-\frac{h}{2}\right)=0 \\
A_{2}\left(\frac{h}{2}\right)=0 & \frac{d A_{2}}{d z}\left(\frac{h}{2}\right)=0
\end{array}
$$

\section{Numerical examples}

\subsection{Analysis of simply supported FGM beam under sinusoidal pressure}

Let us consider a simply supported FGM beam subjected to a sinusoidal pressure as shown in Fig. 3. For convenience, the results are indicated in the coordinate system of Fig. 3 [6]. The Young's modulus is assumed to be of the form $E=E_{0} e^{a z / h}$ and the constitutive equation is the same as that of Ref. [7].

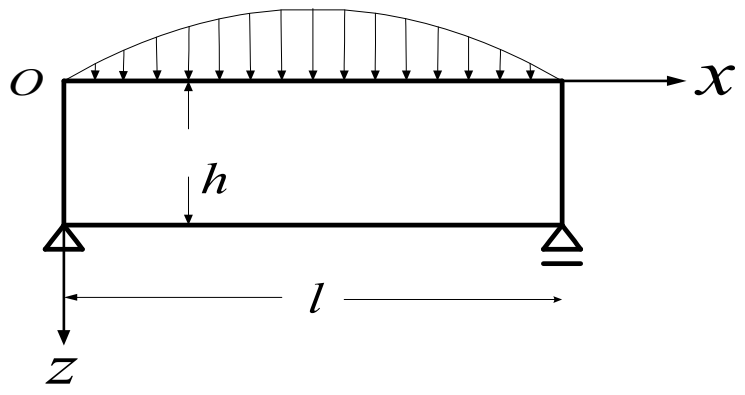

Figure 3: A simply supported FGM beam subjected to sinusoidal pressure.

Two types of the material properties are considered, and the ratios of Young's moduli of the top surface and the bottom surface are $E_{h} / E_{0}=10$ and $E_{h} / E_{0}=0.1$. In the case of the former, the graded index $\alpha$ in Eqn. (5) corresponds to $\alpha=+2.30$, and in the case of the latter, it corresponds to $\alpha=-2.30$. Further, in the case of the former, the load is applied on the softer 
surface and in the case of the latter, on the harder surface. In both cases, Young's modulus is $E_{0}=1$ [Gpa], Poisson's ratio is $v=0.25$ and the thickness is $h=10$ [mm]. The type of the applying force is sinusoidal $p_{z}(x)=p_{0} \sin \xi x=p_{0} \sin \mathrm{i} \pi x / l, \quad \xi=i \pi / l, i=1,3,5 \cdots$.

Figure 4 shows the axial stress distribution at the middle span for $E_{h} / E_{0}=10, \xi h=1$, and Fig. 5 shows the shear stress distribution at the middle span for $E_{h} / E_{0}=0.1, \xi h=3$. In the figures, "Galerkin method" indicates the results of Ref. [6]. The vertical axis is the normalized stress values. The stress $\sigma_{x x}$ is divided by the $\sigma_{x x}(x, h)$, and the stress $\sigma_{x z}$ is divided by the average value at the middle section. From these results, it can be noted that the present results agree well with the results by Ref. [6] for the various conditions of the analysis. Analysis of cantilever FGM beam under uniform pressure

The analysis of the cantilever FGM beam subjected to the uniform pressure as shown in Fig. 2 have been carried out [9]. The length $l$ is $l=1[\mathrm{~m}]$ and the thickness $h$ is $h=0.2[\mathrm{~m}]$. The material properties at $\mathrm{z}=\mathrm{z}_{0}=0$ are given Table 1 , and the magnitude of the transverse pressure $p_{0}$ is $p_{0}=1[\mathrm{~Pa}]$.

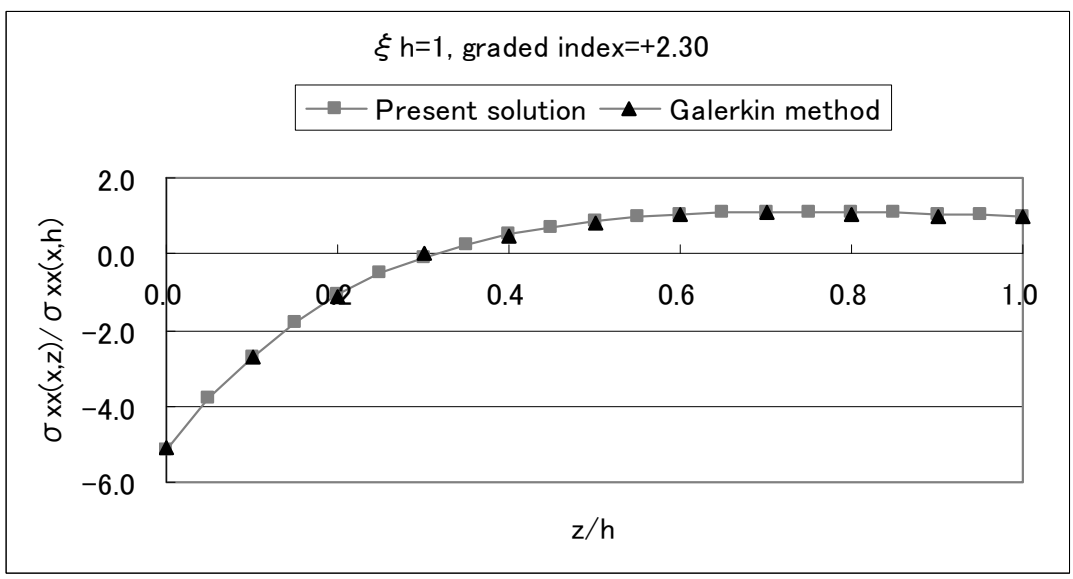

Figure 4: Axial stress $\sigma_{x x}(x, z) / \sigma_{x x}(x, h)$ through the thickness of FGM beam at $x=l / 2$.

For the graded index $\alpha= \pm 3$, Fig. 6 shows the distributions of the stress component $\sigma_{x x}$ at the clamped edge. The horizontal axis is the non-dimensional coordinate $z$ and the vertical axis is the stress values. In this figure, "Ref. [9]" means the results of Ref. [9]. The present results again are in good agreement with those of Ref. [9]. It was also confirmed that the distributions of the stress components $\sigma_{z z}$ and $\sigma_{z x}$ are also in good agreement. 


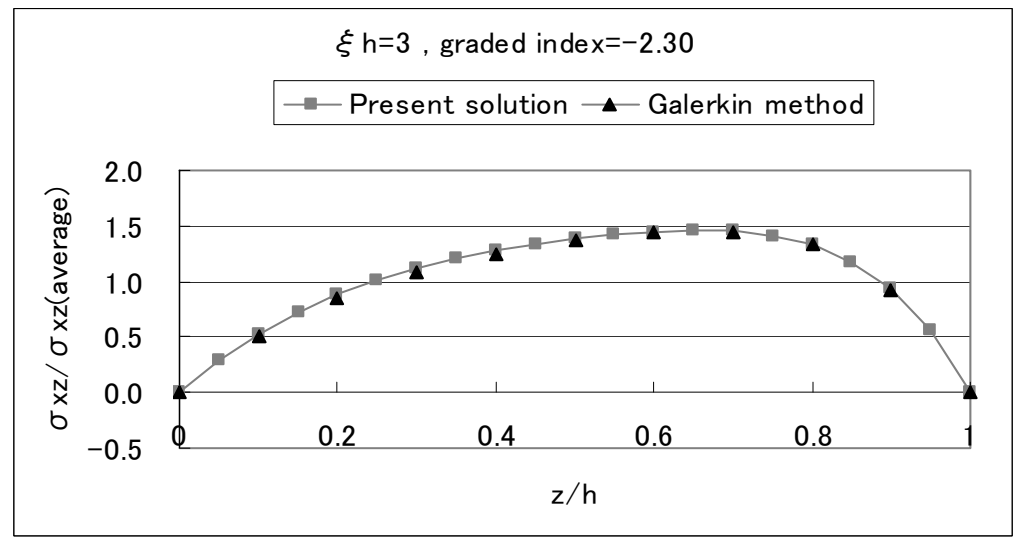

Figure 5: Shear stress $\sigma_{x z} / \sigma_{x z}$ (average) through the thickness of FGM beam at $x=l / 2$.

Table 1: $\quad$ Materials properties.

\begin{tabular}{|c|c|}
\hline Material constants & Values $[1 / \mathrm{Pa}]$ \\
\hline$s_{11}^{0}$ & $5.41 \times 10^{-12}$ \\
\hline$s_{13}^{0}$ & $-1.51 \times 10^{-12}$ \\
\hline$s_{33}^{0}$ & $9.52 \times 10^{-11}$ \\
\hline$s_{44}^{0}$ & $1.37 \times 10^{-10}$ \\
\hline
\end{tabular}

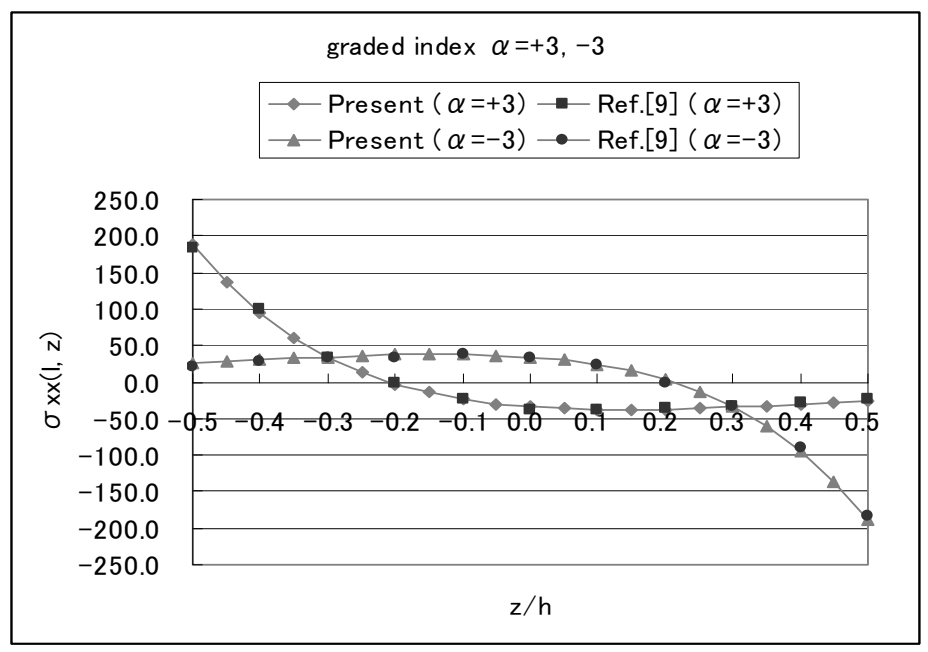

Figure 6: $\quad$ Stress at the clamped end $\sigma_{x x}(l, z)(\alpha= \pm 3)$. 


\subsection{Analysis of simply supported FGM beam under uniform pressure}

The simply supported FGM beam as shown in Fig. 1 is analyzed. The sizes, the elastic constants and the applied load are the same as those of the previous section. The graded index $\alpha$ is assumed as $\alpha=+3$. The distributions of the stress components $\sigma_{x x}$ at the $x=1 / 2=0.5$ and $\sigma_{z x}$ at the $x=0$ are plotted in Figs. 78. The number of terms adopted in the Fourier series of Eqns. (9) and (14) is ten. We confirmed that taking at least eight terms is sufficient accuracy for the solution in this problem.

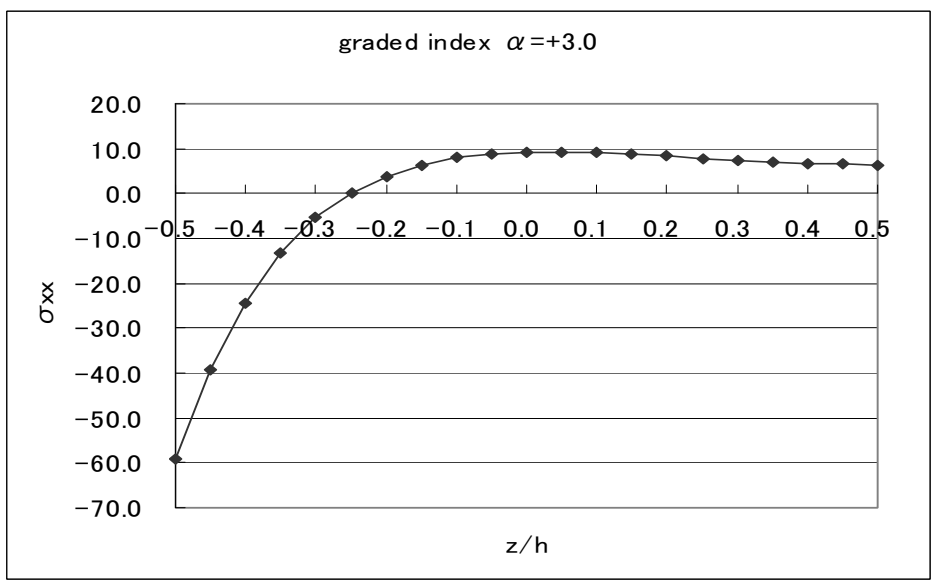

Figure 7: $\quad$ Stress $\sigma_{x x}$ through the thickness at $x=0.5$.

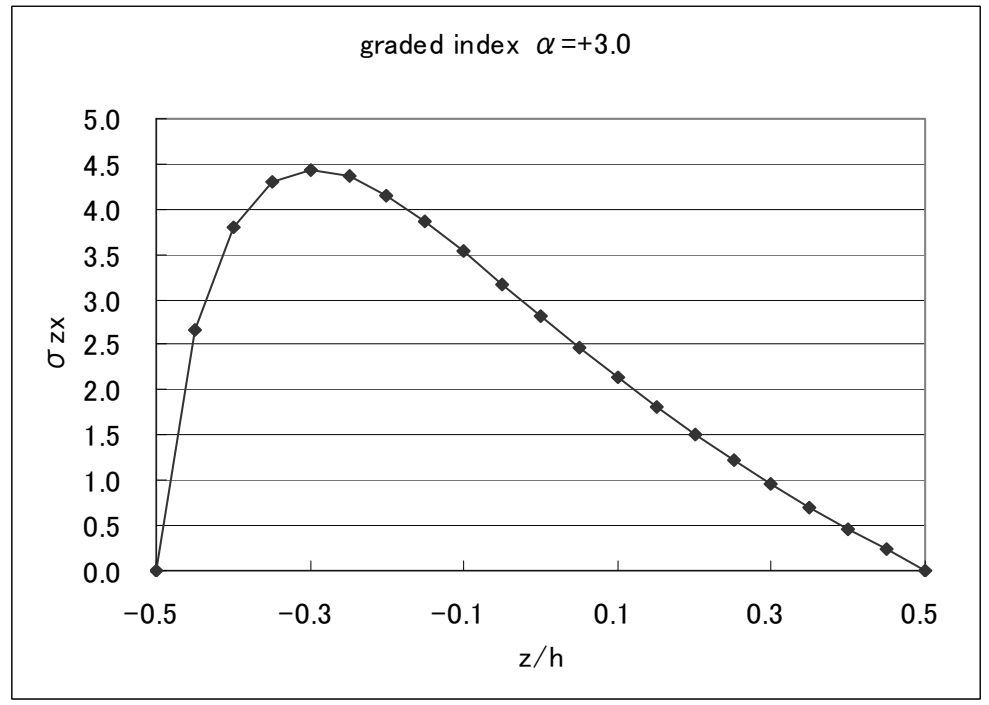

Figure 8: $\quad$ Stress $\sigma_{z x}$ through the thickness at $x=0$. 
From these results, the value of the stress value $\sigma_{x x}$ is greater than that of the stress $\sigma_{z x}$. Comparing the tensile stress $\sigma_{x x}$ on the bottom surface with the compressive stress $\sigma_{x x}$ on the top surface, the greater compressive stress is caused on the softer surface. The shear stress distribution $\sigma_{z x}$ is different from that for homogeneous beams. The point where the maximum stress appears moves toward the softer surface. It may be possible to control distribution of stresses by adjustment of materials properties.

\section{Concluding remarks}

In this paper, one analytical solution method for two-dimensional elastostatic problems in FGMs in which the properties of the materials vary exponentially in one direction is proposed. The method is based on the idea that the governing equation of this problem is of the same form as the governing equation for the plate bending with variable rigidity. The analysis method uses the Airy stress function. From analysis of a few numerical examples, most of the results correlate well with other solutions and the validity of the method is shown. The distributions of displacement components are not stated, however we can obtain them by integrating the strains and displacements relationships.

Future work will be focused on development of an analytical method of solution for analyzing problems of FGMs having an arbitrary variation of their material properties expressed by functions other than exponential.

\section{References}

[1] Suresh, S., and Moretensen, A., Fundamentals of Functionally Graded Materials, IOM Communications Ltd, London, 1998.

[2] Chakraborty A., Gopalakrishnan S. and Reddy JN., A new beam finite element for the analysis of functionally graded materials, International Journal of Mechanical Science, Vol.45, pp.519-539, 2003.

[3] Trung-Kien Nguyen, Karam Sab and Guy Bonnet, First-order shear deformation plate models for functionally graded materials, Composite Structures, 83, pp.25-36, 2008.

[4] H.J. Xinag and J. Yang, Free and forced vibration of a laminated FGM Timoshenko beam of variable thickness under heat conduction, Composites: Part B Vol.39, pp.292-303, 2008.

[5] Sankar BV., An elasticity solution for functionally graded beams, Composite Science Technology, 61, pp.689-696, 2001.

[6] Zhu H. and Sankar BV., A combined Fourier series-Galerkin method for the analysis of functionally graded beams, Journal of Applied Mechanics, Vol.71, pp.421-424, 2004. 
[7] Sankar BV. and Tzeng JT., Thermal stresses in functionally graded beams, AIAA Journal, Vol.40, pp.1228-1232, 2002.

[8] L.S. Miers and J.C.F. Telles, Two-dimensional elastostatic analysis of FGMs via BEFM, Engineering Analysis with Boundary Elements, Vol.32, pp.1006-1011, 2008.

[9] Zheng Zhong and Tao Yu, Analytical solution of a cantilever functionally graded beam, Composite Science and Technology, Vol.67, pp.481-488, 2007.

[10] Mansfield, E.H., The Bending and Stretching of Plates, Pergamon Press, pp.64-73, 1964. 